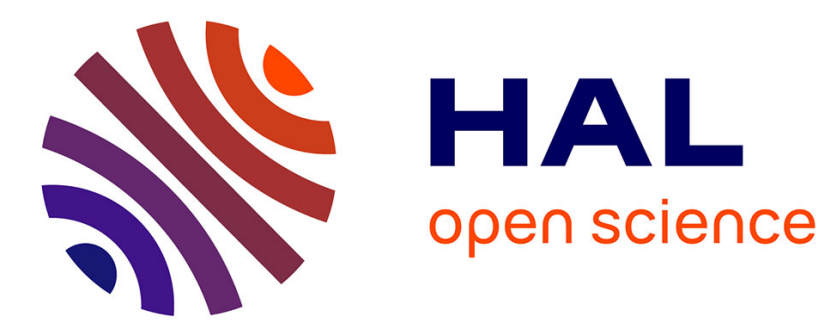

\title{
Faire face aux imprévus sans être pris au dépourvu: le cas des chefs de culture dans de petites entreprises horticoles
}

\author{
Valérie Zara-Meylan
}

\section{- To cite this version:}

Valérie Zara-Meylan. Faire face aux imprévus sans être pris au dépourvu: le cas des chefs de culture dans de petites entreprises horticoles. Sociologies pratiques, 2013, 26, pp.41-56. 10.3917/sopr.026.0041 . halshs-00864244

\section{HAL Id: halshs-00864244 \\ https://shs.hal.science/halshs-00864244}

Submitted on 20 Sep 2013

HAL is a multi-disciplinary open access archive for the deposit and dissemination of scientific research documents, whether they are published or not. The documents may come from teaching and research institutions in France or abroad, or from public or private research centers.
L'archive ouverte pluridisciplinaire HAL, est destinée au dépôt et à la diffusion de documents scientifiques de niveau recherche, publiés ou non, émanant des établissements d'enseignement et de recherche français ou étrangers, des laboratoires publics ou privés. 


\section{Faire face aux imprévus sans être pris au dépourvu : le cas des chefs de culture dans de petites entreprises horticoles}

Valérie Zara-Meylan, ergonome, chercheure au Centre d'Études de l'Emploi, Gis CREAPT ${ }^{1}$

Article paru dans : Sociologies pratiques, 2013/1 n 26, p. 41-56. http://www.cairn.info/revue-sociologies-pratiques-2013-1-page-41.htm

Comment faire face aux imprévus sans être pris au dépourvu? Cette question est au cœur du métier de chef de culture que nous avons observé au cours d'interventions ergonomiques dans de petites entreprises de l'horticulture ornementale. En mettant en évidence des configurations redoutées par des chefs de culture dans leur activité, nous analysons les modalités par lesquelles ils gèrent et anticipent les risques pour leur santé et celle des ouvriers qu'ils encadrent. Cette analyse permet de comprendre que certaines contraintes qui sembleraient inévitables peuvent pourtant faire partie du champ des transformations de l'organisation du travail.

Dans les petites entreprises, les questions de santé liées au travail sont particulièrement difficiles à sortir de l'ombre. C'est le cas du secteur de l'horticulture ornementale où, selon les commanditaires de nos recherches appartenant à des organismes publics, paritaires et professionnels de ce secteur (cf. Encadré), les difficultés économiques persistantes semblent prioritaires, reléguant ainsi au second plan des préoccupations qui concernent la santé des salariés. Cette mise en invisibilité est encore accentuée par de profondes mutations de l'ensemble de ce secteur, dans lequel un mouvement d' "industrialisation » des systèmes de production a été engagé pour faire face à ces difficultés.

Les dirigeants des entreprises participant aux études avaient effectivement insisté, dans leurs demandes, sur leurs inquiétudes concernant la fragilité économique de leurs structures. Dans un marché devenu très concurrentiel, ils faisaient état de «retours qualité » menant à des pertes de clients, sans qu'ils aient plus d'information de la part de ces clients sur leurs critères pour ces retours. Malgré une modernisation et une mécanisation de leurs équipements, ils déploraient aussi des difficultés de recrutement et de fidélisation des permanents comme des saisonniers, en s'interrogeant sur les évolutions du métier qui auraient dû selon eux rendre au contraire leurs entreprises plus attractives. Une autre difficulté qu'ils mettaient en avant était une complication croissante des affectations aux tâches, car certains salariés ne peuvent plus manipuler les plantes les plus couramment produites suite à des restrictions médicales d'aptitudes limitant leur port de charges. Ils estimaient ces difficultés comme liées à «un déficit de compétences » de leurs salariés, en particulier à certains postes jugés clés tels que celui de chef de culture.

\footnotetext{
${ }^{1}$ Centre de recherche sur l'expérience, l'âge et les populations au travail 
Ainsi, un enjeu de notre intervention a été de construire une visibilité sociale des problèmes de santé et de leurs relations avec le travail (Gollac \& Volkoff, 2006) dans ces petites entreprises, à partir de l'analyse de l'activité des chefs de culture. En mettant en évidence leurs compétences déployées dans la gestion des situations qu'ils redoutent, l'objectif a été d'ouvrir des possibles pour transformer l'organisation, compte tenu des exigences productives et de la structure de leur main d'œuvre.

\section{1- Un rapport aux risques du métier : entre euphémisation et naturalisation}

Une relative mise à distance des problèmes de risques pour la santé dans les demandes initiales peut sembler paradoxale, mais elle n'est pas étonnante, en elle-même, et cela pour plusieurs raisons. D'une part, les conditions du travail agricole sont réputées pour leurs contraintes prédéterminées et inévitables, liées à l'environnement dit naturel. «La terre est basse, quoi », ou encore "la pluie, j'ai l'habitude », récapitulait un chef de culture. De plus, comme l'expliquait un autre, les sollicitations physiques répétitives semblent constituer l'inévitable face cachée du "progrès » que constitue la mécanisation partielle du travail : "Quand on doit déplacer 10000 plantes, pendant trois jours on est devant un tapis. Ce sont les risques à payer ». Ainsi, l'horticulture, pourtant traversée par un courant de modernisation des systèmes de production, n'échappe pas à une représentation naturalisante et fataliste des risques du métier.

D'autre part, il est courant que, dans les petites entreprises, une situation financière précaire amène à occulter la nécessité d'aborder des problèmes d'atteinte à la santé, et ce d'autant que les risques peuvent sembler liés à "des activités telles que tout un chacun peut les mener » dans l'espace domestique et donc privé, et ils n'ont pas la «noblesse d'estime» de " grands risques industriels de la grande entreprise » (Verdier, 2010, p. 41). Pourtant des enquêtes nationales montrent un fort taux d'accident ou de maladie professionnelle, d'une part dans les petites entreprises, et d'autre part en agriculture, où une tendance à la dégradation des conditions de travail est établie (Béguin \& Pueyo, 2011). Ces conditions sont caractérisées, en horticulture, par des combinaisons de sollicitations physiques, de contraintes d'ambiances (dans les serres, les tunnels, en extérieur), et d'exposition à des agents biologiques et chimiques. Les exigences organisationnelles d'une forte saisonnalité s'y ajoutent, avec une attente de la hiérarchie en termes de flexibilité des travailleurs de production, souvent en charge aussi des expéditions.

Dans les entreprises de ce secteur, les chefs de culture sont chargés du suivi des plantes et de l'organisation du travail de production. Ils ont un rôle clé dans l'atteinte des objectifs de production, tout en étant garants du contrôle des risques, à la fois pour les plantes et pour les ouvriers. Pour remplir leur mission, ils déploient un travail d'organisation (de Terssac, 2002) qui consiste à interagir avec de multiples acteurs internes (le dirigeant en tant que supérieur hiérarchique, le commercial et la logistique) ou externes à l'entreprise (les fournisseurs et les clients). Les logiques de ces acteurs sont diverses souvent en tension. Elles sont à articuler avec les exigences multiples de la conduite technique de la production. Ce travail 
d'organisation est central, mais invisible aux yeux des dirigeants, ce qui leur donne le sentiment d'avoir «à se débrouiller». Ils sont pourtant aux prises avec des écheveaux temporels emmêlés qui peuvent être sources d'effets disparates et souvent négatifs, à la fois sur la production et la relation aux clients (Dujarier, 2006), ainsi que la santé (Volkoff, 2008).

À partir d'analyses de l'activité de chefs de culture, notre intervention met en lumière différentes composantes des situations qu'ils ont à gérer et leurs conséquences, dans ces systèmes sociotechniques complexes et dynamiques, marqués par les imprévus et les risques.

\section{2- Les « configurations redoutées » : quels risques ?}

Nous proposons une approche ergonomique fondée sur des analyses de l'activité réalisées auprès de cinq chefs de culture dans plusieurs entreprises (cf. Encadré). La relative ancienneté de ceux que nous avons rencontrés dans ces petites entreprises peut s'expliquer par des anticipations et des régulations leur permettent de tenir, bon an mal an, à ces postes, et de produire en limitant les risques. Plus encore, l'analyse de leur gestion de configurations qu'ils «redoutent» particulièrement montre que les chefs de culture expérimentés tentent de reprendre la main sur les dynamiques techniques et sociales des situations.

Encadré : Une méthode fondée sur l'analyse ergonomique de l'activité

Cette analyse est fondée sur des recherches menées par une équipe d'ergonomes du Gis Créapt ${ }^{2}$, au cours de deux projets ${ }^{3}$ en partenariat avec des acteurs de la prévention des risques professionnels, des responsables des politiques de formation et des animateurs de la fédération professionnelle du secteur de l'horticulture (Zara-Meylan, 2012). Dans cet article, nous présentons des analyses concernant cinq chefs de culture, ayant 7 à 35 ans d'ancienneté dans quatre entreprises de 4 à 24 permanents. Elles sont de taille courante ou plutôt grande dans un secteur où la moyenne est de 4,7 équivalents temps plein (Viguier, 2006). Chaque chef de culture a en charge la production sur un site et l'organisation du travail de 2 à 6 ouvriers permanents, 1 à 2 apprentis et jusqu'à 3 saisonniers.

Dans chaque entreprise, l'intervention s'est déroulée sur une période de deux à six mois. Après une phase d'entretiens avec les dirigeants, puis la consultation de documents sur l'organisation, les évolutions récentes et les indicateurs de santé au travail, nous avons mené des entretiens semi-dirigés sur les relations santé travail avec des salariés de tout niveau hiérarchique. Il faut souligner que l'expression de leurs difficultés participait, pour les travailleurs, à une mise en visibilité de problèmes jusque-là difficiles à faire entendre (Molinié, 2010), dans le cadre de projets dont les objectifs de transformation ouvraient une brèche dans une supposée inéluctabilité des contraintes.

Des entretiens plus spécifiques avec les chefs de culture ont ensuite cherché à aborder les conditions temporelles de leur activité et les « configurations redoutées ». Une attention particulière a été portée

\footnotetext{
${ }^{2}$ Centre de recherche et d'études sur l'âge et les populations au travail.

${ }^{3}$ L'un est le projet Equal Agriquadra, mené en partenariat avec le Fonds national d'Assurance Formation des Salariés des Exploitations et entreprises Agricoles (FAFSEA), la Caisse Centrale de la Mutualité Sociale Agricole (CCMSA) et, pour l'un de ses quatre volets sectoriels, avec la Fédération Nationale des Producteurs de l'Horticulture et des Pépinières (FNPHP), de 2006 à 2008. Le second projet a été mené en partenariat avec la Fédération des MSA (mutualités sociales agricoles) du Languedoc en 2009 et 2010.
} 
à la grille de questionnement qui a visé à comprendre les difficultés et les risques du point de vue de leur travail, en considérant ces travailleurs comme producteurs de sens (Demazière \& Dubar, 2004).

Puis nous avons réalisé des observations systématiques de l'activité des chefs de culture durant une à quatre journées, à des périodes ciblées avec eux pour la possible survenue de dynamiques redoutées, et en les complétant par des cahiers de suivi. Comme il est de règle en ergonomie de l'activité, leurs commentaires, enregistrés pendant leur travail ou au vu de nos résultats, ont été incorporés à l'analyse.

Dans cette démarche, ces analyses s'appuient donc sur les entretiens et les observations pour établir des catégories d'interprétation, sans préjuger de classes, relatives à des rapports sociaux par exemple, qui auraient été pré élaborées dans d'autres situations.

Nous examinons tout d'abord, dans les entreprises étudiées, les différentes exigences temporelles enchevêtrées qui peuvent compliquer leur travail. Puis, nous étudions plus spécifiquement l'activité des chefs de culture à partir des «configurations redoutées », un concept inspiré du "vol redouté » introduit par Claude Valot (1998) pour analyser l'activité métacognitive des pilotes en aéronautique : «Ce qui est redouté par l'opérateur, et source de complexité subjective, est de se voir entraîné dans un domaine de moindre compétence où il serait confronté à des aspects du contrôle de ce processus qu'il ne serait pas en mesure de maîtriser » (p. 44). Dans notre objectif, il s'agit d'identifier des configurations redoutées pour leurs dynamiques discordantes, qui selon les chefs de culture, peuvent avoir des conséquences en termes de risques à la fois pour la production et pour la santé.

\subsection{Les composantes multiples des exigences du travail en horticulture}

La production horticole engage de multiples variétés de produits vivants et périssables ${ }^{4}$, qui constituent de nombreux processus dynamiques à conduire sur des espaces délimités (parcelles extérieures, tunnels ou serres), dans des conditions qui ne sont qu'en partie modulables, même sous abri. Cette production est destinée à des ventes échelonnées, dans des systèmes sensibles à la saisonnalité et aux variations évènementielles.

Alors que pèsent des coûts et règlementations alourdis et une concurrence accrue (Viguier, 2006), nombre d'entreprises ont engagé une importante modernisation. Elle se traduit par une mécanisation de certains soins aux plantes (irrigation, gestion climatique, rempotage...), et par l'introduction d'aides à la manutention et par l'aménagement d'abris, par exemple pour la préparation des expéditions. Dans le même temps, une bureaucratisation associée à une rationalisation du travail se traduisent par l'introduction de systèmes de gestion commerciale, de procédures de suivi et de contrôle du travail avec la mesure de cadences lors de certaines opérations de mise en culture et de soins aux plantes. Cependant, notre étude montre que, loin de garantir une régularité de la production et de soutenir la réactivité prônée par la nouvelle

\footnotetext{
${ }^{4}$ Citons par exemple les 600 variétés, réparties en 1200 lots à conduire au même moment, début juillet, dans une grande pépinière (cf. Encadré ci-après) positionnée sur une large gamme de plantes de haie, à destination de jardineries et de paysagistes.
} 
politique commerciale orientée client, la rationalisation ne semble pas non plus simplifier les conditions de la gestion temporelle dans l'activité. Elle semble au contraire multiplier et densifier des dynamiques déjà particulièrement complexes et imbriquées.

\subsection{Les atteintes à la santé révélées par certaines exigences du travail}

L'analyse des données des entreprises horticoles et les premiers entretiens avec les salariés montrent de nombreux problèmes de santé. Prenons le cas d'une grande pépinière employant vingt-quatre permanents (cf. Encadré), trois apprentis et cinq à sept saisonniers au printemps et en été, et dans laquelle cinquante-six accidents avec ou sans arrêt de travail ont été déclarés dans les trois années précédentes (Pueyo \& Zara-Meylan, 2012). Outre de nombreuses lésions (atteignant surtout mains et doigts, et parfois les bras et la tête), des contusions et des atteintes articulaires (lombalgies et membres supérieurs) ont été à l'origine de soixante deux jours d'arrêts de travail. Deux restrictions d'aptitude au port de charge ont même été déclarées dans l'année. La situation est d'autant plus alarmante qu'au cours des entretiens, douze permanents, cinq saisonniers et deux apprentis de 17 ans déclarent des douleurs articulaires.

Dans cette pépinière, les travailleurs associent ces douleurs aux cadences exigées : «j'ai mal à l'épaule. Quand ça va vite c'est bien beau mais le lendemain on a du mal à se remettre » explique une ouvrière (38 ans). Comme nombre de travailleurs des autres entreprises, ils sont concernés une prescription et un contrôle du travail fondés sur des «temps de travaux » pour différents types d'opérations, réalisées sous cadence d'une machine ou entièrement à la main. Ces indicateurs ont été établis dans chaque entreprise pour comptabiliser le nombre d'unités, par exemple rempotées, taillées, tuteurées, désherbées ou expédiées. En imposant une répétitivité à un rythme soutenu et prolongé, ils peuvent s'avérer en eux-mêmes facteurs de risques pour la santé articulaire. De plus, ils ne tiennent pas compte des conditions qui peuvent compliquer le travail (pluie, volume du feuillage, épines, etc.). Ainsi, les travailleurs s'interrogent sur leurs possibilités de «tenir physiquement», disent-ils, dans ce secteur. D'autant qu'ils sont parfois amenés à renoncer à la qualité telle qu'elle est attendue par la hiérarchie, ce qui peut ensuite leur être reproché. Cette situation ébranle leur attachement au métier. «On est des manœuvres en fait », regrette un ouvrier qui ne comprend plus les modes d'évaluation de son travail.

Ces exigences rigidifiées qui révèlent des atteintes à la santé s'inscrivent de façon contradictoire dans une organisation heurtée. Ces exigences sont à examiner dans leurs combinaisons complexes avec d'autres dynamiques de l'organisation du travail.

\subsection{Les dynamiques de configurations redoutées : des discordances à gérer}

Les configurations redoutées par les chefs de culture relèvent de dynamiques complexes dont nous avons analysé des composantes organisationnelles. Nous en présentons trois, pour leurs différentes combinaisons d'enjeux de production et de santé, à comprendre dans des dynamiques aux échelles variées.

\section{a- Des ordres de commande distillés au coup par coup, en ignorant l'incertitude climatique}


"Le plus que je redoute, c'est l'arrachage ». C'est en ces termes que ce chef de culture (54 ans, 10 d'ancienneté), explicite les difficultés de la longue période de vente d'arbres de pleine terre (platanes, hêtres, charmes, etc.) dont il a la charge. Avec son équipe de quatre permanents et deux saisonniers, il ne peut les arracher que l'hiver, lorsqu'ils sont en repos végétatif. Cependant, c'est aussi une période de pluies parfois prolongées. Il explique : "Quand on avance avec l'arbre sur le dos et que les bottes restent plantées dans la boue, ce n'est pas possible de travailler. Mais ils [au service commercial] insistent : il me le faut il me le faut. " Il en précise la dynamique et les conséquences en ajoutant : "S'il a plu pendant quinze jours (...) c'est la pénibilité, c'est la boue. Il nous est arrivé de planter trois machines dans la journée. "

Cette configuration associe les caractéristiques de la période de vente et des articulations avec le responsable commercial dont les exigences sont toujours impératives et distillées de façon quotidienne, sans véritable prise en considération des effets de ces exigences (cf. Figure 1).

Figure 1- Une configuration redoutée dans une pépinière : un arrachage hivernal quotidien, dans des conditions climatiques difficiles

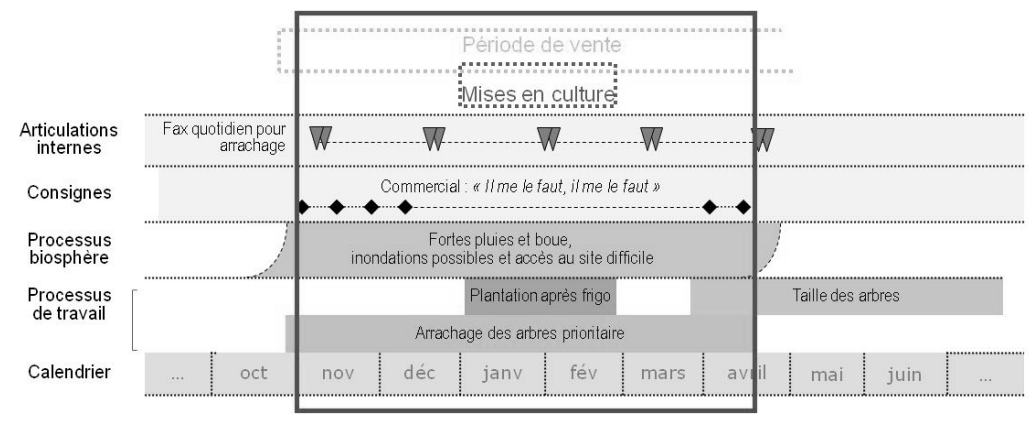

Lecture des figures : La configuration redoutée est comprise dans une dynamique d'évolutions (en abscisse) de différents cadres temporels (en ordonnée) menant à des conjonctions particulièrement critiques à gérer (cadre foncé).

Les risques mis en évidence concernent :

- une «pénibilité » redoutée, car participant à la fatigue accumulée et aux douleurs articulaires dont il fait état pour lui-même et l'équipe, et qui peut avoir des effets sur leur santé,

- des machines mises à rude épreuve, et dont certaines pièces peuvent «casser » sur un terrain devenu difficile,

- les ventes qui peuvent être retardées avec des conséquences commerciales et financières.

Le chef de culture tente d'anticiper la survenue de ces configurations et de modifier leurs conditions par des actions techniques et des interactions sociales. Il gère les parcelles, d'une part en homogénéisant les plantations de jeunes arbres, et d'autre part en arrachant ceux destinés à la vente de manière à dégager peu à peu des passages pour l'accès des machines. Afin d'éviter des réparations difficiles dans la boue et pénalisantes pour l'avancée de l'arrachage, il assure une surveillance et une maintenance préventive des machines. Il 
entretient des relations cordiales avec le commercial pour tenter d'obtenir un planning à la semaine afin de répartir les arrachages selon les conditions biosphériques. Pour appuyer sa demande, il tente d'exposer à la hiérarchie, basée sur un autre site, les différents risques et en particulier leurs conséquences possibles pour les ventes. Cependant, les accords avec le commercial restent verbaux, et toujours à renouveler. N'ayant pu leur donner un statut de règle pérenne et validée par la hiérarchie, il se trouve quotidiennement tenu de faire face à des ordres de commande au coup par coup, auxquels il ne sait plus qu'opposer.

\section{b- Une dynamique de débordement prévisible, ancrée dans l'organisation passée}

Un autre chef de culture (38 ans, 7 d'ancienneté), en charge d'arbustes de haie en pots, avec deux permanents, un apprenti et deux saisonniers, décrit une dynamique au premier abord inéluctable : "Quand les hivers sont cléments, c'est aussi pour les mauvaises herbes ». Puis il précise que cette situation prend son ancrage dans des retards passés : «Parfois, certaines plantes qui n'ont pas bien poussé, qu'on n'a pas taillées, se sont fait envahir». Les conséquences sont redoutables selon lui : "Après vous avez des racines énormes, très dures à arracher. Et même quand vous arrachez, d'autres prennent le relais. (...) Après, vous n'arrivez pas à inclure le désherbage dans tout ce qui est resté à faire. "

La configuration qu'il décrit (cf. Figure 2) associe des conditions climatiques d'un hiver doux à une croissance des herbes favorisée par des retards sur les soins aux plantes (par exemple, un laurier ou un photinia non taillé se dégarnit à la base, ce qui laisse place aux herbes dans le pot). Après de multiples travaux de fin d'hiver, les ventes redémarrent au printemps au moment où il doit aussi mettre en culture de nouvelles plantes. Dans cette période déjà bien chargée s'imposent alors des travaux supplémentaires de désherbage, parfois multipliés suite à ces retards accumulés sur les soins.

Figure 2- Une configuration redoutée : une dynamique de débordement par le désherbage

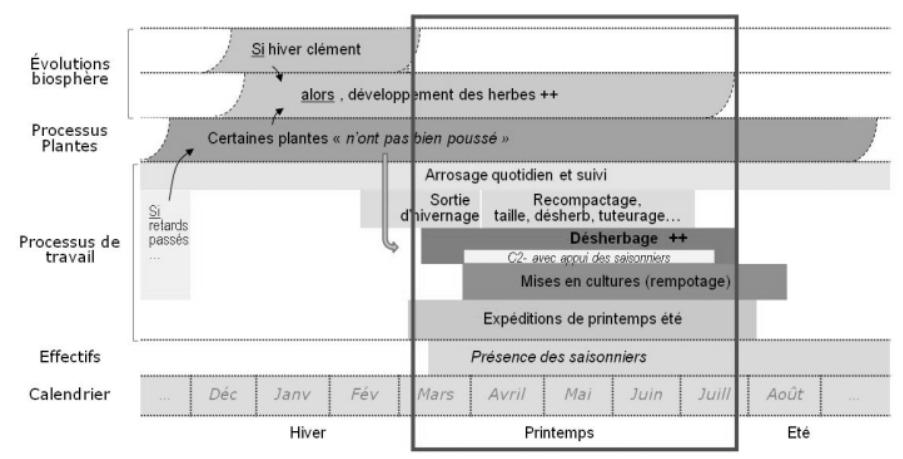

Cette dynamique se solde par des risques qui concernent :

- $\quad$ une production dont la qualité se dégrade au cours de l'hiver,

- une fatigue supplémentaire pour toute l'équipe liée au désherbage qui génère des blessures (éraflures, piqûres), et des douleurs (doigts, genoux, dos),

- un sous-effectif lié au fait que les saisonniers, essentiellement affectés au désherbage ne peuvent plus aider aux mises en cultures et aux expéditions, 
- une situation de débordement en raison d'un écart croissant entre ce qui est à faire et ce qu'il parvient à réaliser.

Le chef de culture tente d'anticiper cette configuration en effectuant régulièrement des soins (taille, tuteurage, désherbage...) «un jour de chaque», même s'il indique que le travail déborde toujours de ces créneaux. Par des questions et des remarques auprès du dirigeant telles que «Les bambous, on ne va pas faire aussi du 6 litres ? Parce que l'an dernier...», il alerte sur les conséquences d'une multiplication des variétés en petits lots qui complique la gestion des soins, et participe au retard. Il tente aussi d'influer sur les décisions de recrutement : "Vous avez prévu quoi, pour les saisonniers? 》 ou encore «Ces herbes, je ne sais pas si on va s'en sortir... », insiste-t-il, en cherchant à maintenir les périodes d'emploi des saisonniers alors qu'elles ont tendance à se rétrécir. Puis, au moment des ventes, il sélectionne les plantes présentant le meilleur aspect pour la vente et le moins de travail pour lui (non taillées et pas envahies). Enfin, lorsque le désherbage s'avère incontournable, il tente d'en limiter l'impact direct sur la santé : "le désherbage, on ne le fait jamais trois jours d'affilée ». Mais cette relative limitation des sollicitations articulaires concerne surtout les permanents. Comme dans nombre d'entreprises, «on attend les saisonniers pour désherber», explique-t-il. Alors qu'il a identifié cette dynamique de débordement, il n'a pas les moyens de l'enrayer, ni d'éviter cette gestion sur le mode du rattrapage.

Les conséquences sur la qualité de la production et sur la santé de tous sont pourtant marquées. De plus les saisonniers, particulièrement sollicités, deviennent difficile à fidéliser d'une année à l'autre. Ainsi, des nouveaux, qui parfois ne restent pas toute la saison, sont à former chaque année. Ce qui renforce au final la tendance à les affecter au désherbage, car les autres tâches telles que la mise en pots et les expéditions nécessitent de savoir-faire plus longs à élaborer.

\section{c- De brusques et régulières exigences de vente, suite à des choix commerciaux et techniques}

En se plaçant à l'échelle d'une journée de printemps, ce même chef de culture fait état d'une tout autre configuration, celle des «fameux lundis du printemps, où les ventes arrivent toutes d'un coup. Là on est pris dans l'urgence». Face à une concurrence accrue, l'enjeu commercial est fort : "On reçoit toutes les commandes et elles sont énormes, et il faut les faire... ». Et dans l'après-midi, le travail se densifie : "En mars, ça tombe vite, la nuit. Et on ne peut plus se poser de question, après. On ne peut plus tergiverser, on ne peut plus réfléchir. (...) On les voit se superposer. Et là, je cours, je cours. C'est interminable, c'est fatigant $»$.

Cette configuration associe les conditions temporelles particulièrement serrées. Les commandes, mêmes tardives, sont impératives du fait d'un affichage commercial réactif de l'entreprise. Et, en l'absence d'éclairage approprié, la tombée de la nuit constitue une butée. De plus, les expéditions nécessitent des réflexions pour une organisation des tournées (avec choix des plantes), des préparations et des conditionnements dans des délais de plus en plus 
restreints. Les commandes tardives amènent parfois à réorganiser le chargement pour changer de conditionnement dans le camion, souvent en limite de capacité. Il faut alors travailler dans l'urgence et en dépassement d'horaire.

Cette situation résulte de pressions externes à l'entreprise dans un secteur très concurrentiel. Elle découle aussi de choix internes, et en particulier d'une politique commerciale prônant la réponse aux commandes de dernière minute, ainsi que de choix techniques (camion à capacité limitée, peu d'éclairage) et organisationnels (livraisons essentiellement le lundi).

Figure 3- Une configuration redoutée : des ventes impératives et tardives d'un lundi de mars

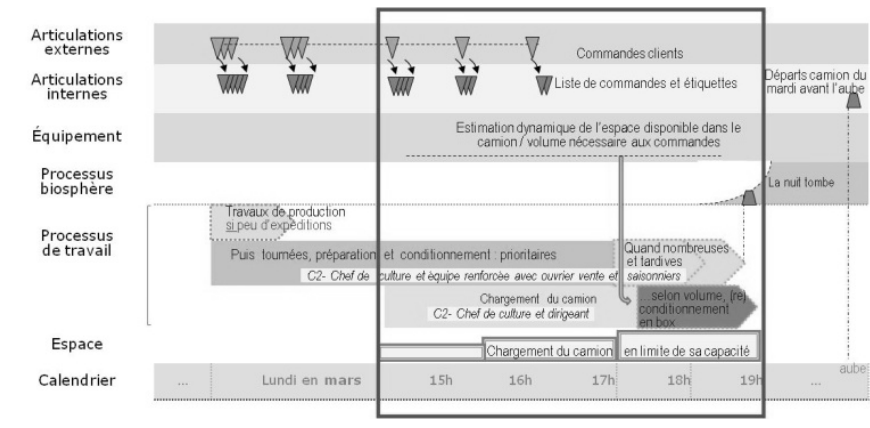

Les risques qui en résultent concernent :

- des oublis possibles dans les préparations en raison du travail dans l'urgence,

- des plantes abimées lors du chargement (plantes entassées, froissées, et parfois cassées durant le transport),

- une fatigue et des douleurs dorsales qu'il a déjà ressenties et qui pourraient ressurgir, en raison des efforts accumulés sur cette période.

Le chef de culture tente d'accroitre sa visibilité sur les commandes envisagées en interrogeant régulièrement la responsable commerciale. Cependant, il n'obtient que peu d'informations. Et dans certaines phases de son travail, il ne peut parfois que s'adapter. Il cherche alors à « gérer son effort " pour tenir, au fil de l'arrivée des commandes tout en réalisant un travail de qualité, et cela au prix d'un fort engagement physique de sa part. Dans cette situation, il explique aussi qu'il cherche à limiter ce qu'il exige des ouvriers, car leur fatigue pourrait peser sur la suite du travail, durant la semaine.

Les configurations redoutées par les chefs de culture prennent leurs ancrages dans des dynamiques tourbillonnaires marquées par la montée de l'immédiateté et l'urgence. Plus largement, les analyses montrent qu'elles voient disparaitre l'indispensable temps-durée (Bergson, 1959, 1ère édt 1907), pourtant nécessaire au travail d'organisation dont ils ont la charge. En tant que responsables de la production et d'une équipe, une autonomie leur est imposée, mais sans que le temps pour réfléchir et organiser ne leur soit accordé, et sans que les informations et le champ d'anticipation (planning de culture, promotions et ventes envisagées, etc.) dont ils ont besoin ne soit reconnus comme relevant de leurs compétences. De plus, ils n'ont pas les moyens de discuter et d'élaborer de règles pérennes. Cette situation est à rapprocher d'un double isolement que nous avons constaté. 
D'une part, étant seuls à exercer cette fonction sur chaque site de production, ils n'ont que très peu d'occasions d'échanger avec des pairs. Les espaces de discussions autour du métier semblent presque inexistants dans ces petites entreprises, d'autant que les référentiels de qualification décrivant leurs missions et attendus n'ont été que récemment établis ${ }^{5}$ et sont encore peu connus dans les entreprises, chaque dirigeant établissant le contour de ce poste en fonction du travail qu'il souhaite déléguer à ce responsable, et de ce qu'il estime de ses compétences.

D'autre part, un second facteur d'isolement est la position d'entre-deux (Dietrich, 2009) qu'ils ont à tenir dans la chaîne hiérarchique, entre la direction et les ouvriers qu'ils encadrent. Dans ces petites entreprises, face à la hiérarchie dont les logiques financières et gestionnaires immédiates priment le plus souvent, ils cherchent à faire entendre les contraintes, exigences et risques du travail qu'ils sont chargés de suivre et d'organiser sur le long cours. Ils sont aussi relativement isolés face aux équipes. Alors qu'ils participent largement au travail de production et déploient un fort engagement physique pour limiter celui attendu des ouvriers, les relations avec eux se tendent. Car faute de marges de manœuvre pour leur travail d'organisation, et dans l'objectif de remplir leur mission de production, ils n'ont parfois d'autre choix que de répercuter les ordres d'immédiateté et d'urgence qu'ils reçoivent, sur les consignes délivrées.

\section{3- Ouvrir des possibles pour une construction sociale des risques pour la santé}

En prenant pour objet les configurations redoutées par des chefs de cultures expérimentés, notre étude a permis de montrer comment les chefs de culture gèrent des exigences sociotechniques qui semblent inéluctables. À l'instar des pilotes d'avion, ils ont parfois le sentiment d'être pris dans des dynamiques dont ils pourraient perdre le contrôle. En effet, leur action sur la qualité des plantes peut être réduite, car étroitement liée aux conditions de culture tout au long du cycle de croissance de chaque lot. Ils n'ont guère d'influence auprès des commerciaux pour négocier des délais. Ils sont peu écoutés par la direction qui semble s'arranger du fait que les chefs de culture se « débrouillent » plutôt que de chercher à articuler une politique commerciale avec des moyens techniques, des effectifs à mobiliser et souvent à former, et les conditions saisonnières, incertaines et variables, mais plus ou moins prévisibles.

L'analyse de ces configurations montre qu'ils font alors parfois des compromis en faveur de la production et au détriment de la santé. Elle montre aussi et surtout que les chefs de culture cherchent tout de même à influer sur ces exigences imbriquées, multiples et parfois discordantes. Pour permettre et appuyer cette gestion, leur travail devrait faire l'objet de suivis, de coordinations entre la production et le commercial, de consultations régulières entre

\footnotetext{
${ }^{5}$ Le référentiel de qualification du métier de chef de culture en pépinière conçu par l'Anefa (Association nationale pour l'emploi et la formation en agriculture) date de 2006, ceux établis par l'AFPA décrivant les métiers d'ouvriers de pépinière et horticole de 2006 et 2007.
} 
ces deux niveaux afin de prendre en considération l'évolution de la qualité des plantes, les conditions techniques et climatiques.

Après validation et accord des chefs de culture concernés, les configurations redoutées ont constitué des supports pour mettre en visibilité leur travail d'organisation auprès des dirigeants et discuter des risques mis en évidence dans l'activité, afin d'envisager des transformations. Dans la pépinière de pleine terre par exemple (cf. ci-dessus, cas a-), suite à une restitution en présence d'une conseillère en prévention des risques professionnels (un partenaire du projet de recherche), de la direction, du commercial et du chef de culture, un planning d'arrachage sur quinze jours a été mis en place, avec des compléments hebdomadaires en fonction des nouvelles commandes. Cette formule s'est avérée compatible, selon la direction, avec la stratégie commerciale et les relations tissées avec les clients.

Dans l'autre pépinière (cas b- et c-), la restitution a abouti à fixer une priorité d'intervention : permettre au chef de culture une gestion plus sereine de l'échelonnement des travaux essentiels en limitant l'accumulation de retards, en premier lieu avec un projet de réaménagement de la zone de rempotage accompagné par le conseiller en prévention. Autour de ce projet, une réflexion plus large sur l'organisation des principaux travaux sur un mode chantier a été engagée, en prévoyant la programmation, la préparation du matériel, des outils et une anticipation des affectations, ainsi que les recrutements nécessaires. Dans cette entreprise, il est aussi envisagé de filtrer les commandes de fin de journée. En fonction des clients et de leurs exigences, un report partiel de la livraison pourrait leur être proposé, pour mieux répondre à leurs attentes en termes de qualité.

Plus largement, dans chacune des entreprises ayant participé à cette recherche, des pistes d'action portent sur les choix stratégiques ou organisationnels de ces entreprises: positionnement commercial, orientations variétales, systèmes techniques, automatisation, modes de contrôle du travail... En effet, on l'a vu, des demandes tardives et pressantes des clients sont prises en compte par les entreprises, engagées dans une logique de réactivité pour trouver une contrepartie dans une production tout à la fois rationalisée et flexible (Pueyo \& Zara-Meylan, 2012). Pris dans cette double contrainte, les chefs de culture se trouvent alors tenus de courir le risque d'agir, pour aboutir au mieux dans la production, dans des dynamiques qui les emmènent au-delà de la zone des risques qu'ils jugent acceptables pour eux-mêmes et les autres travailleurs.

Ainsi, dans chaque entreprise, un aspect essentiel de la transformation se jouait à travers les évolutions organisationnelles et les mesures techniques engagées. Plus fondamentalement, au cœur de ces discussions sur le travail des chefs de culture et de leurs compétences, c'est en réalité la pérennité de la production et les risques acceptables, du point de vue des différents acteurs de l'entreprise qui étaient mis en débat.

Pour permettre et soutenir les transformations locales, les analyses et les pistes d'actions ont plus largement été présentées et discutées à plusieurs niveaux d'influence et de décision, en particulier au sein de la fédération professionnelle de l'horticulture, dont les animateurs pour certains eux-mêmes dirigeants, orientent la politique sectorielle en influent sur les stratégies 
de gestion et de positionnement commercial. Elles ont aussi été discutées pour faire évoluer des approches de la santé et des risques professionnels dans ce secteur, et dans d'autres petites entreprises, auprès des partenaires projets chargés de la prévention des risques, de la formation professionnelle et des réflexions sur les qualifications.

La mise en visibilité des configurations redoutées et leur mise en débat constitue une proposition pour intervenir auprès d'acteurs à différents niveaux, en contribuant à mettre à la lumière le travail invisible de gestion de ces responsables sommés de «s'en débrouiller ». La prise en compte des risques pour la santé dans l'activité ouvre des possibilités de transformation du rapport des organisations aux risques. Si les dirigeants de petites structures sont aux prises avec l'immédiateté et des urgences qu'ils relayent vers le bas de la hiérarchie, la pénibilité qui a pu jusque-là être considérée comme inéluctable est désormais analysée comme un défaut d'organisation.

\section{Références bibliographiques}

Béguin, P., \& Pueyo, V. (2011). Quelle place au travail des agriculteurs dans la fabrication d'une agriculture durable ? Pistes, 13(1), http://www.pistes.uqam.ca/.

Bergson, H. (1959, 1ère édt 1907). L'évolution créatrice (Vol. Version numérique de Gemma Paquet d'après une édition PUF 1959). Chicoutimi: Cégep.

Demazière, D., \& Dubar, C. (2004). Analyser les entretiens biographiques. Québec: Les Presses de I'Université de Laval.

Dietrich, A. (2009). Le manager intermédiaire ou la GRH mise en scène. Management \& amp; Avenir, 21(1), 196-206.

Dujarier, M. (2006). L'idéal au travail. Paris: PUF.

Gollac, M., \& Volkoff, S. (2006). La santé au travail et ses masques. Actes de la Recherche en Sciences Sociales, 163, 5-17.

Molinié, A.-F. (2010). Queixa. Laboreal, 6(1), 69-71, http://laboreal.up.pt.

Pueyo, V., \& Zara-Meylan, V. (2012). Impacts d'outils de gestion sur la conduite de cultures en pépinière. @ctivités, 9(1), 1-20 http://www.activites.org.

de Terssac, G. (2002). Le travail d'organisation comme facteur de performance. In G. De Terssac (Ed.), Le Travail : une aventure collective. Recueil de textes (pp. 149-159). Toulouse: Octarès.

Valot, C. (1998). Métacognition et connaissances métacognitives. Intérêt pour l'ergonomie. Unpublished Thèse de doctorat de nouveau régime. Spécialité Ergonomie, Toulouse.

Verdier, É. (2010). Risques professionnels : une affaire de générations dans les TPE de la réparation automobile. In CEE (Ed.), Actes du séminaire CREAPT Ages et Travail 2008. Rapport de recherche $C E E n^{\circ} 58$. Expérience professionnelle et gestion de la sécurité au travail (pp. 41-58). Paris: Centre d'Etudes de l'Emploi.

Viguier, M. (2006). Les perspectives économiques des secteurs de l'horticulture. Avis et rapports du conseil économique et social. Paris: Les éditions des Journaux Officiels.

Volkoff, S. (2008). L'intensification disperse les problèmes de santé. In G. De Terssac, C. Saint-Martin \& C. Thébaud (Eds.), Travail, organisation, santé : une question de précarité. Toulouse: Octarès.

Zara-Meylan, V. (2012). Modalités de gestion du milieu temporel dans une conduite de processus multiples en situation dynamique : une recherche dans des entreprises horticoles. Unpublished Thèse de doctorat en Ergonomie, préparée au Créapt, S. Volkoff et $V$. Pueyo (dir.), Cnam, CEE, Noisy-le-Grand. 